\title{
Electric Breakdown Through Nano Dielectric Films
}

\section{S Abdalla*}

Medical Physics, Faculty of Science, King Abdulaziz University, Saudi Arabia

One of the most important candidates of next generation electronics is molecular electronics, as miniaturization of silicon semiconductors reaches its physical and economic bounds. Some studies on electrical transport through nanometer $(\mathrm{nm})$ thick Dielectric Films (DF) are complicated because of local electrical breakdown due to the nm-size characteristics. In order to turn around this problem, one should know the minimal voltage responsible for these $\mathrm{nm}$ breakdowns. This can need more difficult measurements and not easy interpretations. In this article, one can propose and present a new technology to minimize the presence of local breakdown that occurs at the nm level and can present a model system to explain what can happen through DF. In this technology, one can sandwich nano-gold particles (Au-pad) between the gold electrode and DF. Gold nano-particles can play the role of lightning rod effect (or sometimes called lightning conductor). Thus, macro-electrodes can measure currents with ratio nano-particles contacts and thus one ensures the lowest electric breakdown (EBD) voltage of a specific dielectric film. Then, one can use $\mathrm{SiO}_{2}$ thin substrate on which a thin film of about some angstroms monolayer of organic molecules (to link the Au nano-particles). Here, one can measure the breakdown voltage in the range from 1 volt up to 6 volts in two cases: The first when one sandwiches the organic film between the voltage terminals then varying the applied dc-voltage slowly from 1 volt to the critical (breakdown) voltage. The second case, one can measure the EBD voltage when sandwiching gold bads of $5 \mathrm{~nm}$ between the organic film and gold electrode. One expects that net difference between the values of breakdown in the two cases can appear because the larger of gold nanoparticles on the film surface don't necessarily result in significantly higher breakdown voltages. The above mentioned experiments can be repeated once more but one can replace DF by deoxyribonucleic acid DNA molecule. One expects getting new experimental data about the charge transfer through DNA. This shows the need for combining experimental data with significant model. In this presumed model, one can explain the two opposite effects of the particle size, i.e., increase in defect density in the insulator and decrease in the local electric field intensity. This can lead us to more understanding the mechanism of breakdown and would explain why these systems are vulnerable to electrical breakdown as a result of spikes in regular electrical grids. To confirm that the breakdown occurs really in the nm scale, one can take some image of the film surface before and after breakdown.

To enhance developing molecular electronic devices, one should take into account the following:

1- A suitable method to evaluate all properties (chemical and physical properties) and characteristics of single molecular device.

2- A fabrication process suitable to produce molecular devices. Both items should be synchronically carried out. One should take into account the intrinsic nano-breakdown phenomena that take place through nano-materials and correct self-breaking technology should be used in order to keep the material out of damage. Under high field conditions, particularly under DC, electric charges accumulate inside a nano-insulating material which distorts the electric field. This would lead to premature aging (due to accumulation of charges at defects) and failure. Zhao et al. [1] have recently shown that thermally heated nano-wires semiconductors break at the midpoint, while metallic nano-wires breakdown near the two ends due to the stress induced by electro-migration. The different breakdown mechanisms for the nano-wires are caused by the different thermal and electrical properties of the materials.

The need for cheap, flexible, and transparent electrical contacts for optoelectronic applications, in particular photo-voltaic and light emitting diodes, has spurred development for novel methods for preparing such contacts. The resulting contacts, in nanometer (nm) cases, have a high aspect ratio (the aspect ratio is the square of the sample length to its breadth); for example contacts with $\mathrm{nm}$ size play an essential role during the charge transfer through nano-material such as metal nano-wire [1], carbon nano-tubes [2,3], deoxyribonucleic acid DNA molecule [4]. Generally, such contacts serve to study low conductance nm materials.

Early, it has been shown [5] that rough electrical contacts can influence charge transport through a layer of low conductance material by causing Electrical Breakdown (EBD) which if unaccounted for can lead to artifacts in experimental data. This has been attributed to the increased electric fields at certain locations with minimum thickness. High surface aspect ratio contacts would presumably show similar properties as they, too, might lead to enhanced electric fields. This kind of interpretations could allow choosing or modifying high surface aspect ratio contacts to have the desired optical properties, while keeping the EBD-voltage above an acceptable level. Enhanced EBD can also be manifested in experiments such as edge-contacts [6], breakjunctions [7], nanogaps [8], etc. Researches, in these fields, are usually aware of this possibility and try to avoid it for nano-electronic transport measurements. This would be of interest if local current enhancement can help or is the purpose for creating trapped space charges $[9,10]$. The use of very small contact areas and contacts stability issue, for these single junction methods, requires extensive data collection and tedious statistical analysis to determine the minimal breakdown voltages. To look closely into this issue, one can use a structure which is common to researchers and applications, with one of the contacts a conducting substrate, onto which the low conductance layer is deposited, with a high surface aspect ratio contacts and second contact on the other side of the low conductance film (Figure 1). Such model can investigate what can happen with high surface aspect ratio contacts, if one takes into account the following conditions:

a. The substrate should be chemically and mechanically stable, electronically conducting, and as smooth as possible.

*Corresponding author: S Abdalla, Medical Physics, Faculty of Science, King Abdulaziz University, Saudi Arabia, E-mail: smabdullah@kau.edu.sa

Received August 22, 2012; Accepted August 24, 2012; Published August 31, 2012

Citation: Abdalla S (2012) Electric Breakdown Through Nano Dielectric Films. J Material Sci Eng 1:e102. doi:10.4172/2169-0022.1000e102

Copyright: ( $) 2012$ Abdalla S. This is an open-access article distributed under the terms of the Creative Commons Attribution License, which permits unrestricted use, distribution, and reproduction in any medium, provided the original author and source are credited. 


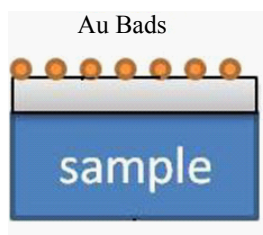

Figure 1: Low conductance film.

b. The film (low conductance layer) should be chemically and physically as one well-defined, and as pin hole free as possible. Reproducible and reliable application of the second contact is possible, with and without nm-sized features.

c. The substrate, the film, and the second contact preparations, all need to be reproducible.

d. While one can study the $\mathrm{EBD}$ of $\mathrm{SiO}_{2}$ with a thin organic monolayer on its top, the system would be adapted easily for other types of inorganic or organic insulators, for metal (or semiconductors) substrate and for high surface aspect ratio particles of different compositions and shapes. Thus, one can use this method to minimize, as possible, EBD properties of high-k insulators, such as aluminum oxide $\mathrm{Al}_{2} \mathrm{O}_{3}$ with high surface aspect ratio contacts. The gold nano particles can play the role of lightning rod effect (or sometimes called lightning conductor) which is expected to reduce the EBD through DF and DNA molecule.

\section{References}

1. Zhao J, Sun H, Dai S, Wang Y, Zhu J (2011) Electrical Breakdown of Nanowires Nano Lett 11: 4647- 4651.

2. Azulai D, Belenkova T, Gilon H, Barkay Z, Markovich G (2009) Transparent Metal Nanowire Thin Films Prepared in Mesostructured Templates. Nano Lett 9: 4246-4249.

3. Wu Z, Chen Z, Du X, Logan JM, Sippel J, et al. (2004) Transparent, Conductive Carbon Nanotube Films. Science 305: 1273-1276.

4. Abdalla S (2011) Electrical conduction through DNA molecule. Prog Biophys Mol Bio 106: 485-497.

5. Bhattacharyya A (1984) Modeling of write/erase and charge retention characteristics of floating gate EEPROM devices. Solid State Electronics 27 899-906.

6. Singha S, Thomas MJ (2008) Dielectric properties of epoxy nanocomposites. IEEE T Dielect EI In 15: 12-23.

7. Tsutsui M, Shoji K, Taniguchi M, Kawai T (2008) Formation and Self-Breaking Mechanism of Stable Atom-Sized Junctions. Nano Lett 8: 345-349.

8. Standley B, Bao W, Zhang H, Bruk J, Lau CN, et al. (2008) Graphene-based atomic-scale switches. Nano Lett 8: 3345-3349.

9. Enkov ET, Palaria A (2004) Charge writing in silicon-silicon dioxide for nanoassembly. Nanotechnology 15: 1211-1216.

10. Ganesan K, Ilango S, Mariyappan S, Baroughi MF, Kamruddin M, et al. (2011) Conductive atomic force microscopy studies on dielectric breakdown behavior of ultrathin $\mathrm{Al}_{2} \mathrm{O}_{3}$ films. Appl Phys Lett 98: 092902. 